\title{
Hydrogen Storage in Iron/Carbon Nanopowder Composite Materials: Effect of Varying Spiked Iron Content on Hydrogen Adsorption
}

\author{
Chun-Lin Chu, ${ }^{1}$ Chia-Feng Chang, ${ }^{2}$ Jiann-Ruey Chen, ${ }^{2}$ and Yiin-Kuen Fuh ${ }^{3}$ \\ ${ }^{1}$ National Nano Device Laboratories, Hsinchu, Taiwan \\ ${ }^{2}$ Department of Materials Science and Engineering, National Tsing Hua University, Hsinchu, Taiwan \\ ${ }^{3}$ Department of Mechanical Engineering, National Central University, Taoyuan County, Taiwan \\ Correspondence should be addressed to Yiin-Kuen Fuh; michaelfuh@gmail.com
}

Received 20 December 2012; Revised 6 February 2013; Accepted 6 February 2013

Academic Editor: Hongmei Luo

Copyright (C) 2013 Chun-Lin Chu et al. This is an open access article distributed under the Creative Commons Attribution License, which permits unrestricted use, distribution, and reproduction in any medium, provided the original work is properly cited.

\begin{abstract}
This study investigates the effects of varying the spiked iron content of iron/carbon nanopowder (Fe/CNP) composite materials on hydrogen storage capacity. Among four such samples, a maximum hydrogen uptake of approximately $0.48 \mathrm{wt} \%$ was obtained with $14 \mathrm{wt} \%$ of spiked iron under $37 \mathrm{~atm}$ and $300 \mathrm{~K}$. This higher hydrogen uptake capacity was believed to be closely related to the physisorption mechanism rather than chemisorption. In this case, the formation of maghemite catalyzed the attraction of hydrogen molecules and the CNP skeleton was the principal absorbent material for hydrogen storage. However, as the iron content exceeded $14 \mathrm{wt} \%$, the formation of larger and poorly dispersed maghemite grains reduced the available surface areas of CNP for the storage of hydrogen molecules, leading to decreased uptake. Our study shows that hydrogen uptake capacities can be improved by appropriately adjusting the surface polarities of the CNP with well dispersed iron oxides crystals.
\end{abstract}

\section{Introduction}

Hydrogen-based energy is an irreplaceable component in the inventory of future energy sources. Hydrogen has long been considered an ideal energy carrier. In addition, it is a viable alternative to fossil fuels such as oil and coal because it is a renewable energy source that causes no carbon emissions [1]. Despite the promise of hydrogen as an abundant energy source, several obstacles such as its production, transportation, and storage need to be overcome in order to make hydrogen-based energy practical and viable. In order to meet the challenges of hydrogen storage, four different storage methods have been proposed: liquefaction, compression, storage in metal hydrides, and adsorption on certain surfaces [2]. Among these currently available techniques, those involving adsorption and the use of a solid-state matrix composed of metal hydrides are considered particularly efficient in mild temperatures [3]. Although metal hydrides can store large amounts of hydrogen through chemisorption, they have high mass densities and unsuitably slow hydrogen desorption rates. In order to address these issues, two methods have been proposed: the doping of transition metals with catalytic properties to lower the desorption energies involved [4] and the selection of light-weight metal hydrides [5].

Microporous carbon-bearing materials have also been considered for hydrogen storage. These materials possess properties that include not only large specific surface areas [6] but also microporous textures [3] that provide a particularly favorable environment for hydrogen storage. These carbonbearing materials adsorb undissociated hydrogen molecules mainly by the van der Waals forces present at their surfaces. These binding forces are so weak that the physisorption process at room temperature is impeded by thermal motion [3]. Transition metals are again introduced into these carbonbearing materials to lower their surface energies to enhance the adsorption forces at room temperature. As a particular example, a report based on the ab initio density-functional theory suggests that pillared Li-dispersed boron carbide 
nanotubes are capable of hydrogen storage capacities of up to $6.0 \mathrm{wt} \%$ [7]. The hydrogen uptake capacities of singlewalled carbon nanotubes can be greatly increased (from $0.76 \mathrm{wt} \%$ to $1.66 \mathrm{wt} \%$ in a particular case) by using doped palladium that has undergone thermal treatment [8]. The use of nickel catalysts in the nanotube material enhances the spillover effect of hydrogen, thereby leading to a higher rate of hydrogen adsorption on the activated carbon surface [9].

In our study, we selected carbon nanopowders (CNPs) spiked with different weight ratios of catalytic iron. Further, we evaluate and discuss the hydrogen storage capacity of these Fe/carbon nanopowder (Fe/CNP) composite materials at $300 \mathrm{~K}$.

\section{Experimental}

2.1. Preparation of Iron/Carbon Nanopowder Composite Materials. The CNP (CAS 7400-44-0) and reagents $\left(\mathrm{Fe}\left(\mathrm{NO}_{3}\right)_{3}\right.$ and an analytical grade of ethanol) were purchased from Aldrich and used without any further purification. The CNP (formed by laser decomposition) was in an amorphous form with an average particle size of $30 \mathrm{~nm}$ (as obtained from the specifications labeled on the bottle). In order to prepare the Fe/CNP composite materials, $0.4 \mathrm{~g}$ of untreated CNP was mixed with $40 \mathrm{~mL}$ of ethanol and the desired amount of $\mathrm{Fe}\left(\mathrm{NO}_{3}\right)_{3}$. The mixtures were sealed by Parafilm and immersed in an ultrasonic water bath for one hour. After the seal film was removed, the samples were dried at $60^{\circ} \mathrm{C}$ for a duration of $24 \mathrm{~h}$.

2.2. Textural and Structural Studies. X-ray diffraction (XRD) analyses (using a LabX XRD-6000, Shimadzu) of the original and iron-spiked CNP samples were conducted with a scan range of $10^{\circ} \sim 80^{\circ}$ (corresponding to 2 theta or twice the incident angle). The scan rate was set at 4 degrees per minute and $\mathrm{CuK} \alpha$ radiation $(\lambda=0.15406 \mathrm{~nm})$ at $30 \mathrm{kV}$ and $20 \mathrm{~mA}$ was used for the diffraction process. The Fourier Transform Infrared Spectroscopy (FTIR) spectra (using a Bomen DA8.3 spectrometer) of the CNP samples were obtained by operating the FTIR in the transmission mode using $\mathrm{KBr}$ pellets with the scan rate and resolution set at $1.00 \mathrm{~cm}^{-1} / \mathrm{s}$ and $2 \mathrm{~cm}^{-1}$, respectively. The transparent $\mathrm{KBr}$ salt pellets were prepared by grinding $5 \mathrm{mg}$ of the $\mathrm{CNP}$ with $50 \mathrm{mg}$ of $\mathrm{KBr}$ salt. Subsequently, this mixture was hydraulically pressed. The specific surface area of the CNP sample was determined using the Brunauer-Emmet-Teller (BET) equation by measuring the $\mathrm{N}_{2}$ adsorption isotherm at $77 \mathrm{~K}$ (ASAP2020, Micromeritics). The pore volume and the pore size of the sample were automatically calculated by the built-in software. The pore volume was determined by the single point adsorption rate for pore diameters less than $62.5368 \mathrm{~nm}$ at $P / P_{0}=0.96807$. The pore size was obtained by the adsorption average pore width $(4 \mathrm{~V} / \mathrm{A})$ evaluated using the BET method.

2.3. Hydrogen Adsorption Experiments. The hydrogen uptake capacity of the CNP samples was evaluated using a specially designed volumetric adsorption/desorption system (shown

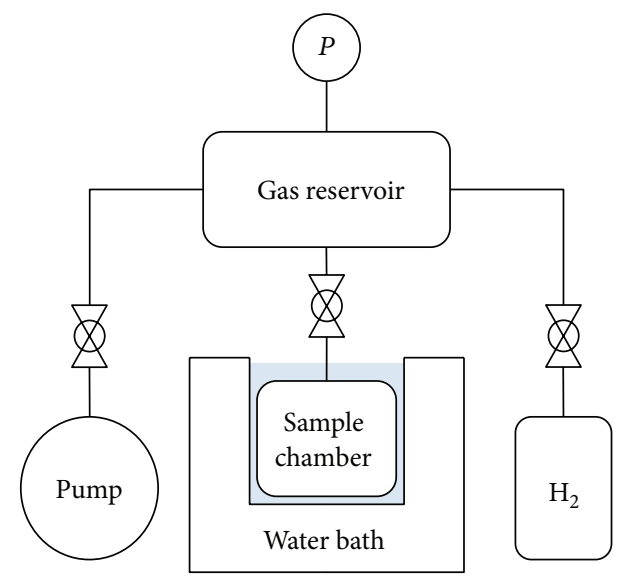

FIGURE 1: Schematic diagram of measurement system of hydrogen adsorption experiments.

in Figure 1). This apparatus comprised a sample cell (with a volume of $10.45 \mathrm{~cm}^{3}$ ) and a gas reservoir cell (with a volume of $30.61 \mathrm{~cm}^{3}$ ) separated by a Sievert valve. The entire system was evacuated and vented several times prior to the experimentation process in order to minimize the hydrogen uptake from the system materials. For the hydrogen adsorption experiments, all the prepared samples were preheated at $360^{\circ} \mathrm{C}$ for $20 \mathrm{~min}$ under 20 atm of pressurized hydrogen. The whole procedure was repeated four times. After the sample cell was pretreated and cooled to room temperature, it was immersed in a water bath in order to be maintained at a constant temperature environment. The hydrogen adsorption experiments were carried out at $300 \mathrm{~K}$ with an initial hydrogen pressure of $37 \mathrm{~atm}$; the pressure drop from this initial pressure was recorded by a pressure gauge. The hydrogen adsorption capacity of the samples was calculated from the pressure drop by using the ideal gas law. The background correction factor for this calculation was obtained by conducting the experiment under identical conditions without using any samples. Subsequently, $\mathrm{LaNi}_{5}$ crystals were used as the internal standard sample and the experiment was repeated.

\section{Results and Discussion}

3.1. Hydrogen Uptake Experiments. The dependence of hydrogen uptake on reaction time under conditions of $37 \mathrm{~atm}$ pressure and $27^{\circ} \mathrm{C}$ (room temperature) is shown in Figure 2(a). Since the steady states of hydrogen adsorption are reached after a reaction time of one hour, the corresponding hydrogen uptake in one hour for all samples is chosen for all the following discussions. As indicated in Figure 2(b), a local adsorption maximum of approximately $0.48 \mathrm{wt} \%$ is obtained with a CNP sample spiked with $14 \mathrm{wt} \%$ of iron. Further, it is noticed that the hydrogen uptake capacity inversely decreases as the iron content increases. This hydrogen storage capacity is comparable with the adsorption capacities of palladiumand vanadium-doped carbon nanotubes ( 0.69 wt\%) [10]. In addition, this storage capacity of the CNP sample is also 


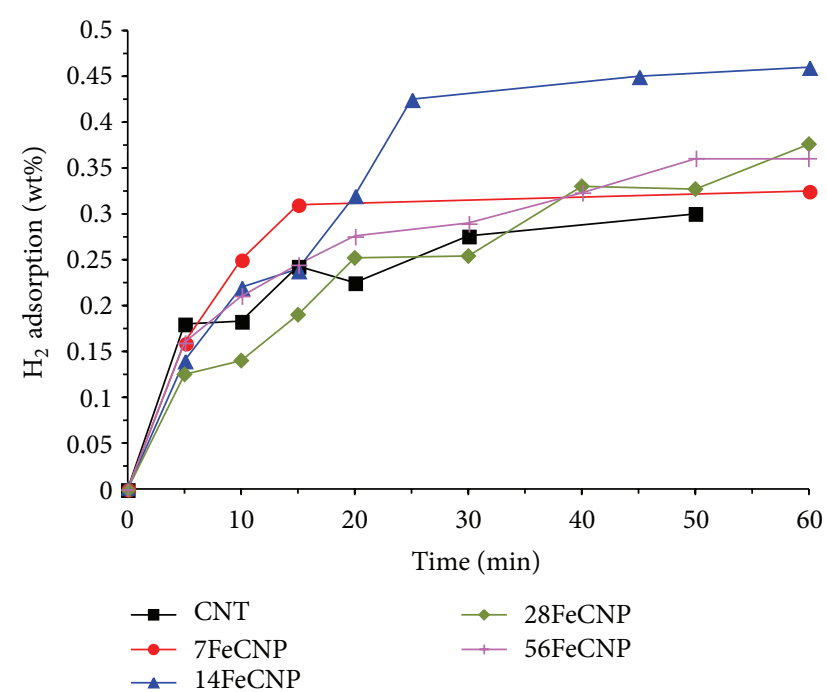

(a)

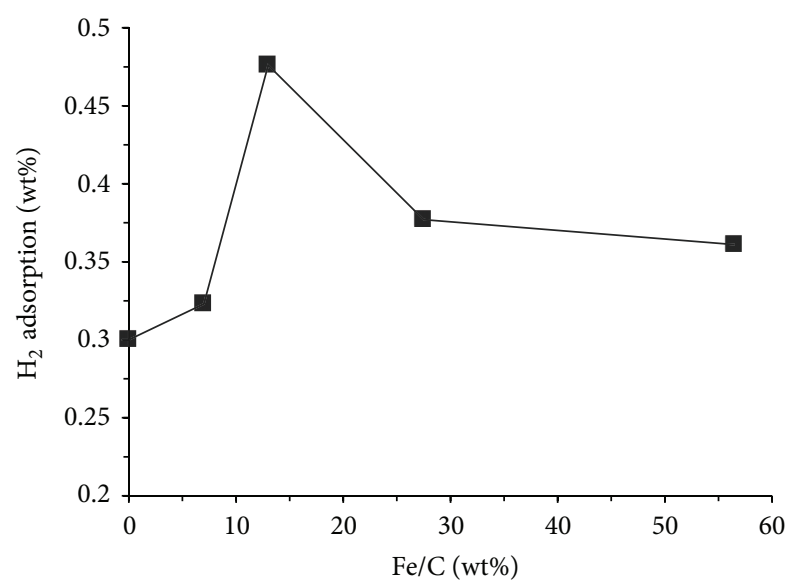

(b)

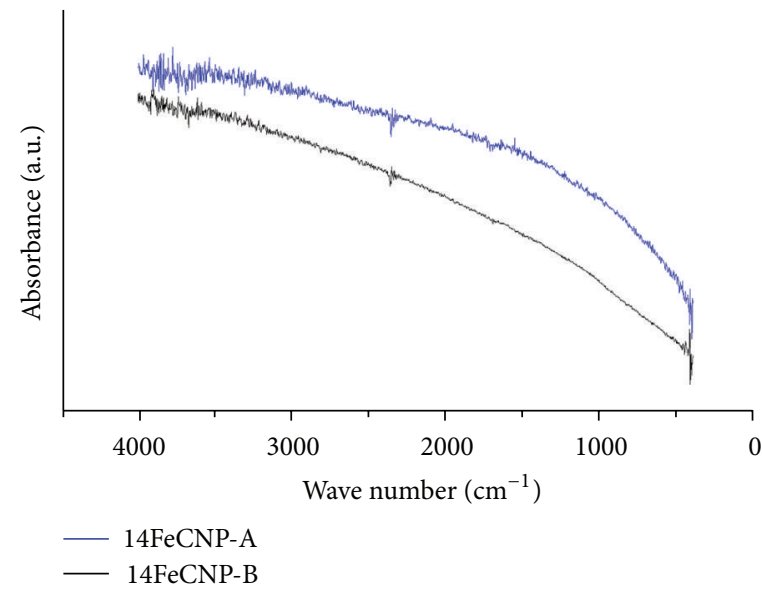

(c)

FIGURE 2: (a) Kinetic energy diagram of hydrogen molecule adsorption to Fe/carbon nanopowder (Fe/CNP) composite materials; (b) effect of iron content in Fe/CNP composite materials on hydrogen adsorption; and (c) FTIR spectra of 14 FeCNP samples before and after hydrogen adsorption.

comparable with that of activated carbon $(\sim 0.53 \mathrm{wt} \%)$ [9]; moreover, it is superior to that of copper-doped activated carbon fibers $(0.28 \mathrm{wt} \%$ under similar conditions) [1]. In spite of the decrease in hydrogen uptake capacity when the spiked iron content exceeds $14 \%$, the overall hydrogen adsorption capacity is enhanced by approximately $20 \sim 60 \%$ in comparison with the original CNP sample that was not spiked with iron. This observation clearly indicates that the addition of iron improves the hydrogen adsorption capacity in carbon-bearing materials.

3.2. Textural and Structural Properties. In order to interpret the differences in hydrogen adsorption capacities of the various samples, the textural and structural properties of the $\mathrm{Fe} / \mathrm{CNP}$ composite samples are examined. Figure 2(c) shows the FTIR spectra of the $14 \mathrm{wt} \%$ iron-spiked samples with the maximal spectral values before (14 FeCPN-B) and after hydrogen adsorption (14 FeCPN-A). After hydrogen is adsorbed in the samples, no $\mathrm{C}-\mathrm{H}$ stretching vibrations are observed. Normally, these vibrations are observed at wavenumber values of approximately $2800 \sim 3100 \mathrm{~cm}^{-1}$ [11]. The suppression of these vibrations might imply either that the amount of adsorbed hydrogen molecules by chemisorptions is not enough to activate the $\mathrm{C}-\mathrm{H}$ stretching vibrations or that there is eventually no chemisorption occurring in the samples.

The specific surface areas of the $\mathrm{Fe} / \mathrm{CNP}$ samples are analyzed by measuring the $\mathrm{N}_{2}$ isotherm at $77 \mathrm{~K}$ using the BET method. Furthermore, these hydrogen uptake capacities are normalized by their corresponding specific surface areas (listed in Table 1 and shown in Figure 3). It is noted that each square meter of surface area contributes to approximately $0.0035 \mathrm{~g}$ of hydrogen uptake; this uptake capacity appears 
TABLE 1: Results of measured $\mathrm{N}_{2}$ isotherm using BET analysis and measured hydrogen adsorption capacities.

\begin{tabular}{lccccc}
\hline Sample & $\begin{array}{c}\text { BET surface area } \\
\left(\mathrm{m}^{2} / \mathrm{g}\right)\end{array}$ & $\begin{array}{c}\text { Pore volume } \\
\left(\mathrm{cm}^{3} / \mathrm{g}\right)\end{array}$ & $\begin{array}{c}\text { Pore diameter } \\
(\mathrm{nm})\end{array}$ & $\begin{array}{c}\text { Gravimetric adsorption capacity } \\
(\mathrm{wt} \%)\end{array}$ & $\begin{array}{c}\text { Normalized adsorption capacity } \\
\left(10^{-3} \times \mathrm{g}-\mathrm{H}_{2} / \mathrm{m}^{2}\right)\end{array}$ \\
\hline $\mathrm{CNP}$ & 110.26 & 0.441 & 15.99 & 0.386 & 3.497 \\
$7 \mathrm{Fe} / \mathrm{CNP}$ & 226.86 & 0.682 & 12.03 & 0.452 & 1.993 \\
$14 \mathrm{Fe} / \mathrm{CNP}$ & 191.13 & 0.429 & 8.99 & 0.610 & 3.191 \\
$28 \mathrm{Fe} / \mathrm{CNP}$ & 128.04 & 0.352 & 11.00 & 0.512 & 3.995 \\
$56 \mathrm{Fe} / \mathrm{CNP}$ & 147.67 & 0.529 & 14.33 & 0.478 & 3.239 \\
\hline
\end{tabular}

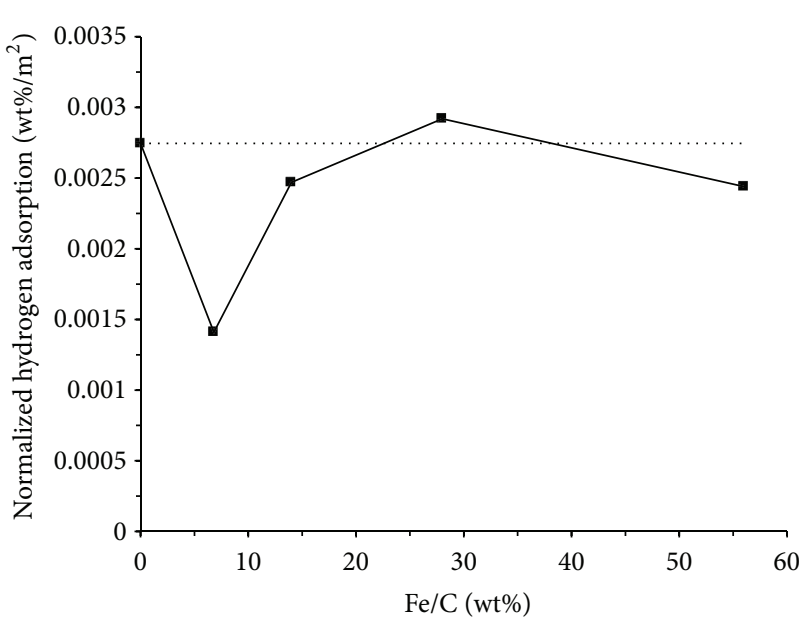

FIGURE 3: Normalized hydrogen uptake rates after considering specific surface areas of Fe/CNP composite materials.

unaffected by the amount of iron present in the sample. Since chemisorbed hydrogen molecules are most likely to occur at the metal surface (metal hydride), the consistent normalized sorption capacity of both the pure and iron-spiked CNP samples might imply that the hydrogen molecules are mainly adsorbed by the process of physisorption. However, the possibility that the hydrogen molecules are adsorbed through the process of chemisorption at the Fe/CNP sample surfaces still exists. Furthermore, it is reasonably assumed that the CNP supports principally contribute to the observed surface areas; the iron-bearing species developed under our experimental conditions usually have a specific surface area of less than $20 \mathrm{~m}^{2} \mathrm{~g}^{-1}$ [12]. Consequently, the presence of iron content would effectively increase the adsorption capacity per surface area of the CNP since the fraction of CNP present in the sample decreases as the iron content increases. However, as observed in Figure 3, the normalized adsorption capacity does not proportionally increase as the iron content increases. This might imply that both the surface morphology and pore distribution of the CNP influence the hydrogen uptake; this influence of the morphology and the pore distribution can be examined by the scanning electron microscopy (SEM) and the $\mathrm{N}_{2}$ isotherm BET analyses.

Figure 4(a) clearly shows that the original CNP is an aggregate of hundreds of granular carbon nanoparticles; this aggregation results in a mesoporous as well as a macroporous texture. This texture is considered an unfavorable environment for hydrogen adsorption. Based upon the grand canonical Monte Carlo calculation, Rzepka and coworkers have suggested that a micropore with diameter of approximately $0.70 \sim 0.75 \mathrm{~nm}$ could effectively retain hydrogen molecules owing to better surface contact (stronger adsorption forces) at the pore walls [3]. In this particular case, the hydrogen molecule resides comfortably in the pore due to the strong capillary action of the adsorption forces. On the other hand, although a larger pore $(>1 \mathrm{~nm})$ could hold more hydrogen molecules by the formation of two adsorption layers, the binding force is so weak that it would be easily overcome by the thermal motion of molecules at room temperature. Accordingly, the dependence of porosity in retaining hydrogen would be limited and the observed gravimetric adsorption would only depend on the specific surface area of the samples [3].

As indicated by the Bopp-Jancso-Heinzinger (BJH) differential adsorption pore volume $(\mathrm{dV} / \mathrm{dD})$ shown in Figure 4(b), the CNP sample mainly contains the mesoporous texture (centered at $2.5 \mathrm{~nm}$ ) and the macroporous surface (centered at $75 \mathrm{~nm}$ ); this is consistent with the aggregated features observed in the SEM image (Figure 4(a)). The addition of iron species would dramatically rearrange the original porous texture toward microporous morphology distribution by the growth or aggregation of Fe-bearing particles (inset in Figure 4(a)). When the iron content exceeds $14 \mathrm{wt} \%$ (14 Fe/CNP samples), the CNP surfaces are fully covered by Fe-bearing particles; thus, the micropores are blocked and the surface texture tends toward mesoporous environments due to the formation of large iron clusters. The addition of iron content increases the overall specific surface area of these Fe/CNP samples to a certain extent; this increase in the specific surface area is responsible for the higher hydrogen uptake. Although the transition images of the porous texture are difficult to record due to the limitations of SEM resolution, the transition in texture could significantly reflect in the $\mathrm{N}_{2}$ BET adsorption/desorption isotherm. Further, this transition can be calculated by the BJH method [13]. However, the increased specific surface area alone cannot fully explain why the maximum hydrogen adsorption does not occur in the $7 \mathrm{wt} \%$ ( $7 \mathrm{Fe} / \mathrm{CNP})$ samples that show the largest specific surface area. In order to address this disparity, it is necessary to take into consideration the role of iron content in the Fe/CNP samples. This is discussed in the following section. 


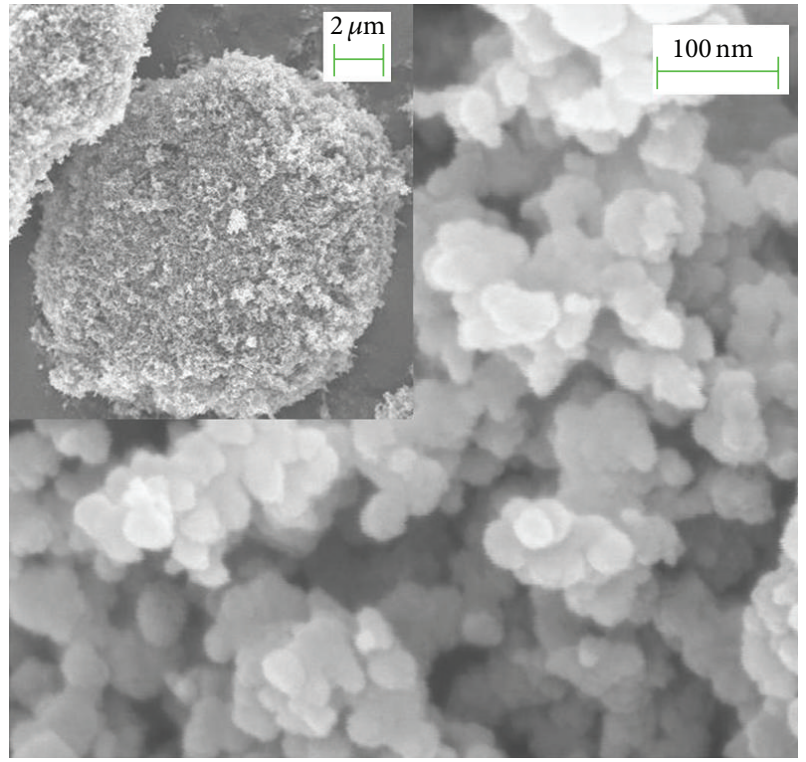

(a)

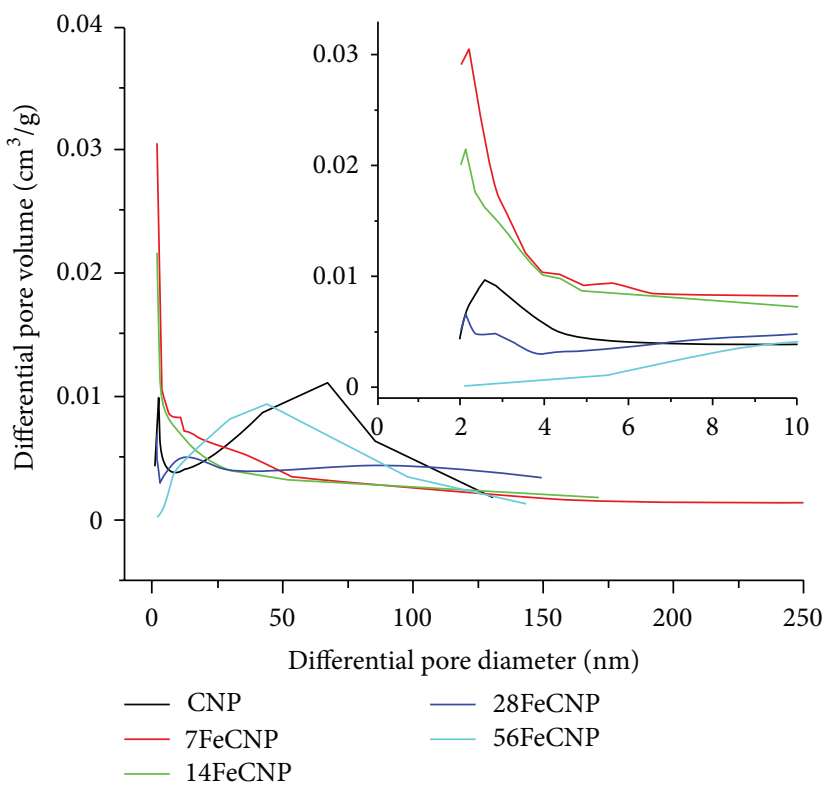

(b)

FIGURE 4: (a) Scanning electron microscopy (SEM) image of original CNP sample; and (b) distribution of pore diameters in Fe/CNP composite materials.
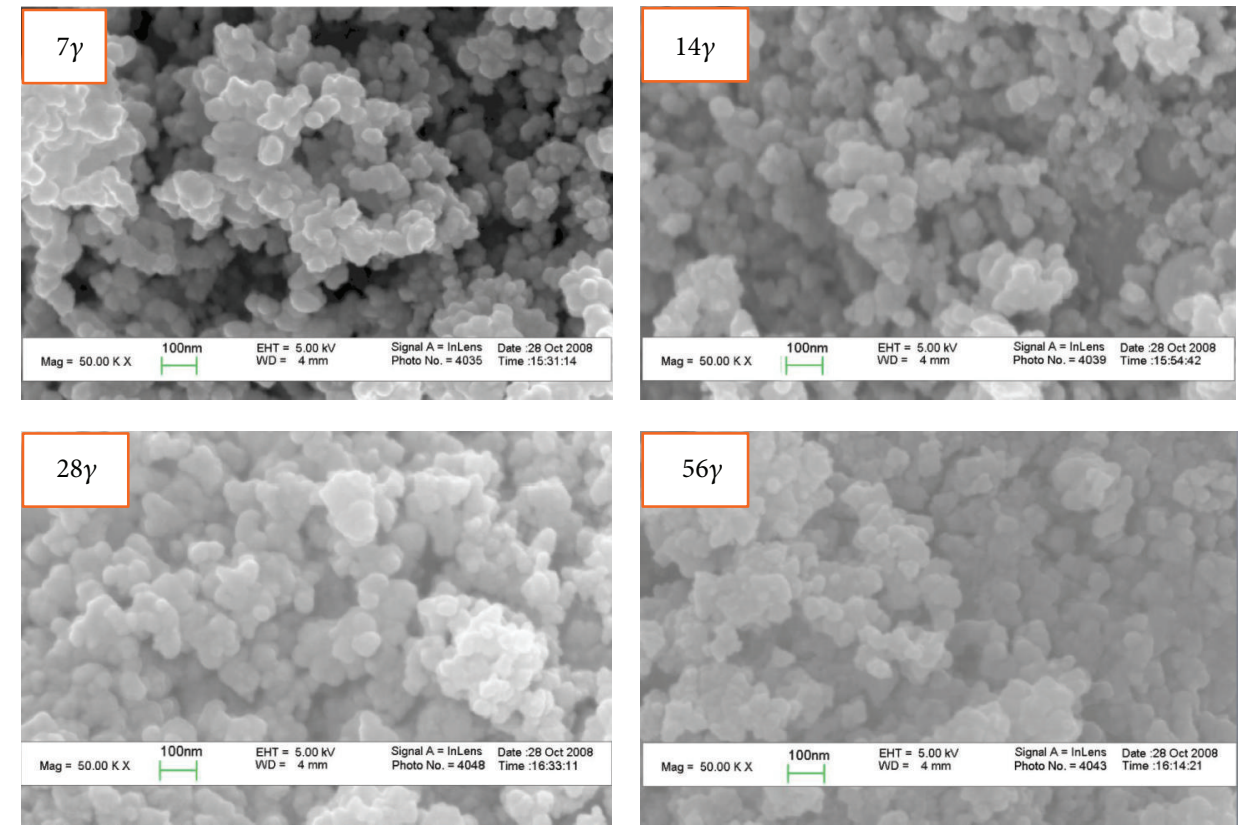

FIGURE 5: Surface morphology of 7\%, 14\%, 28\%, and 56\% $\gamma$ - $\mathrm{Fe}_{2} \mathrm{O}_{3}$ in $\mathrm{Fe} / \mathrm{CNP}$ composite materials.

3.3. Effects of Surface Polarization. Figure 5 shows the SEM image of the formed $7 \%, 14 \%, 28 \%$, and $56 \% \gamma-\mathrm{Fe}_{2} \mathrm{O}_{3}$ in the $\mathrm{Fe} / \mathrm{CNP}$ composite materials. The particles detected in the SEM images have an identical average size as calculated by the XRD line broadening method. A close-up micrograph (Figure 6) shows $\mathrm{Fe}$ in Fe/CNP determined, and Fe should be $\mathrm{Fe} / \mathrm{CNP}$ compound as the SEM/EDS mapping analysis displays the existence of significant $\mathrm{Fe}$ composition. We applied quantitative method of $\mathrm{Fe}$ in $\mathrm{Fe} / \mathrm{CNP}$ determined on those samples (Figure 6). As indicated by the XRD patterns, the spiked iron contents finally transition into a maghemite cluster in the Fe/CNP samples (Figure 7). Maghemite can be considered as a fully oxidized magnetite in which the positive charge is increased via oxidation of $\mathrm{Fe}$ (II) in the magnetite [12]. As a result, it is believed that the surface polarization of Fe/CNP samples (induced by the presence of maghemite) increases as the iron content increases. Polarized surfaces attract hydrogen molecules more effectively than 

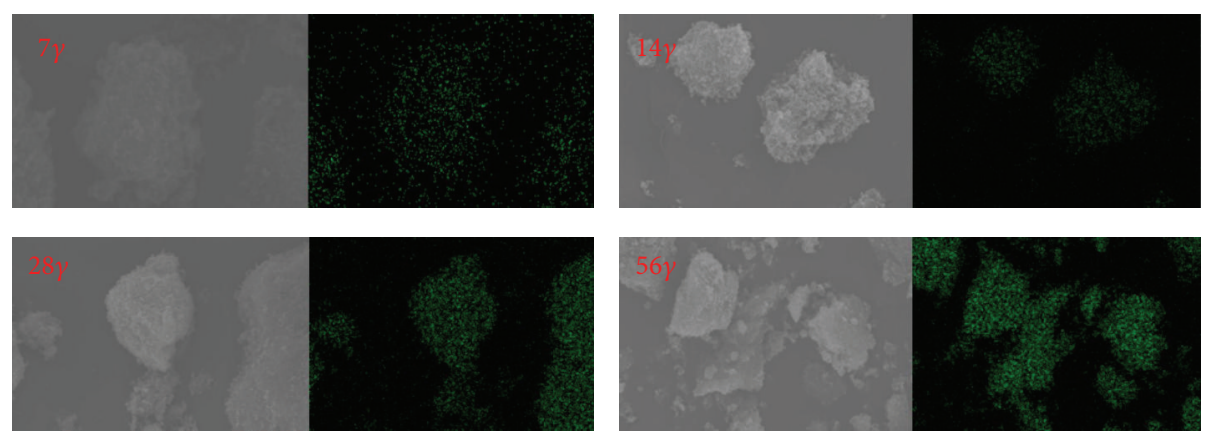

FIGURE 6: Enlarged micrographs with EDS mapping analysis of the Fe in Fe/CNP.

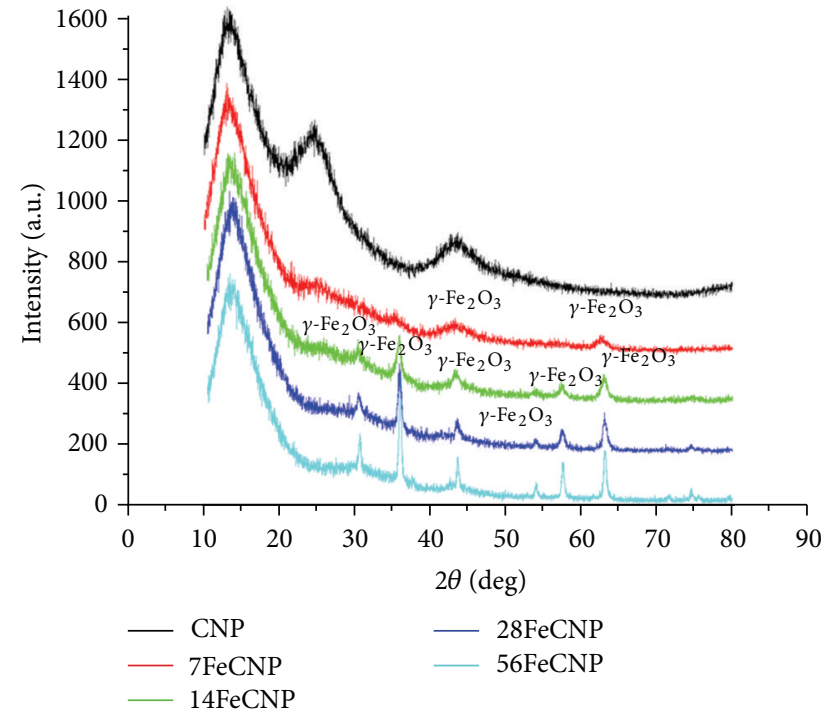

FIGURE 7: X-ray diffraction (XRD) patterns of Fe/CNP composite materials.

relatively neutral CNP surfaces due to the stronger dipoleinduced dipole interactions; in particular, this is observed in the region several angstroms above the sample surface [14]. We have not yet obtained any substantial spectrum evidence to determine whether or not the surface maghemite acts as a catalyst to create the spillover effect of the hydrogen molecules. However, it has been suggested that the initially adsorbed hydrogen molecules would preferentially form a hydrogen layer with the individual hydrogen "atoms" covering the maghemite surfaces [15]. In our study, we have tentatively assigned the formed cluster as maghemite. Further identification work is needed to support our claim.

Under high pressure environments, hydrogen molecules can be adsorbed by the dipole (at the support surface) induced-dipole (partially polarized hydrogen molecules) interactions [1]. At the same time, the nearby carbon supports would also be partially polarized and lose some of their surrounding electrons owing to the strong electron-acceptor characteristic of metallic catalysts. This would create new hydrogen adsorption sites that increase the hydrogen uptake capacities [1]. Consequently, the hydrogen uptake capacity

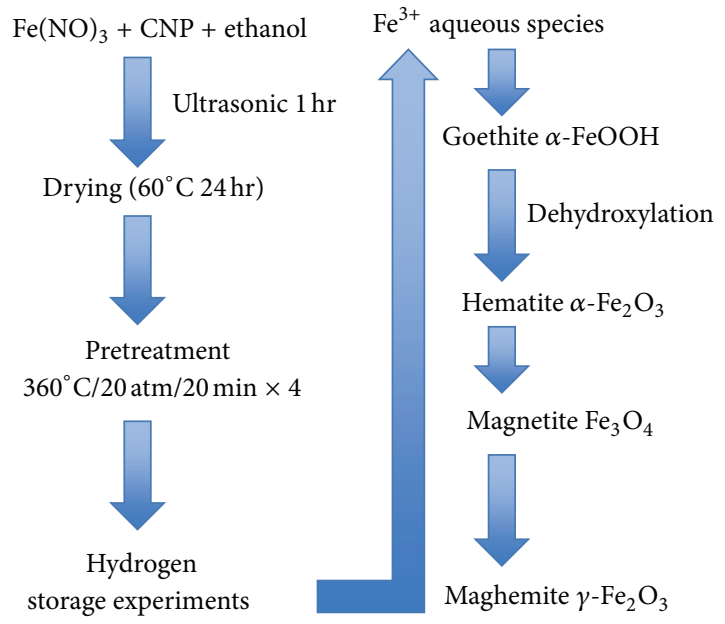

FIGURE 8: Scheme of iron oxide transformation and experimental procedures.

is strongly related to the number of adsorbed hydrogen molecules that overcome the surface energy barrier between the metallic portion and the carbon support [15]. This reasoning more appropriately describes our experimental observations.

During the reactions occurring in the ultrasonic bath, the $\mathrm{Fe}$ (III) ions are able to locate themselves at the CNP surface, while the accompanying water molecules escape during the following $24 \mathrm{~h}$ of drying at $60^{\circ} \mathrm{C}$ (Figure 8). During this stage, we speculate that the Fe (III) ions probably transform into iron-bearing oxide structures; these structures are able to further assist phase transformation to well-ordered iron oxides in the pretreatment stage (Figure 8). In the sample preparation processes as discussed above, the most probable method of maghemite formation is by the dehydration of goethite to form the hematite phase. Subsequently, the hematite is oxidized to form magnetite [12]. The maghemite obtained through this process usually has a specific surface area less than $20 \mathrm{~m}^{2} \mathrm{~g}^{-1}$; this supports our previous reasoning that the CNP content mainly contributes to the measured surface area.

Accordingly, we have presented our speculations on the increased hydrogen adsorption by the Fe/CNP samples in this 
section. In the case of 7Fe/CNP (7 wt\%) samples that exhibit the largest specific surface area, their mild hydrogen uptake capacity might be attributed to the lack of sufficient quantities of maghemite clusters embedded in the CNP skeleton. Under these conditions, only the nearby CNP surfaces are able to adsorb the polarized hydrogen molecules; the rest of the CNP surface contributes very little to the hydrogen adsorption due to the higher surface energy barrier and poor spillover reactions [8]. In addition, this explains the lowest normalized hydrogen adsorption capacity (shown in Figure 3). For the $14 \mathrm{Fe} / \mathrm{CNP}$ samples that show the maximum hydrogen adsorption, the maghemite clusters may probably be well dispersed over the entire carbon nanopowder surface. This arrangement lowers the surface energy barrier but sacrifices the specific surface area since the deposited maghemite might also block the surface pores. Beyond $14 \mathrm{wt} \%$ of the spiked iron sample, further addition of iron species leads to larger maghemite grains on CNP surface; these grains not only block the surface pore (reducing the surface area) but also increase the weight of the Fe/CNP samples. Consequently, the gravimetric storage capacity of these samples drops; however, the normalized adsorption capacity is very close to those of the chosen initial CNP samples.

\section{Conclusion}

In this study, we evaluated the hydrogen storage capacity of $\mathrm{Fe} / \mathrm{CNP}$ composite materials spiked with various weight ratios of iron content. The XRD analysis of the samples showed the formation of maghemite $\left(\gamma-\mathrm{Fe}_{2} \mathrm{O}_{3}\right)$ in these $\mathrm{Fe} / \mathrm{CNP}$ samples. Moreover, no significant chemisorption of hydrogen molecules was observed from the FTIR spectra. The maximum obtained gravimetric hydrogen storage capacity was approximately $0.48 \mathrm{wt} \%$ with a spiked iron ratio of $14 \mathrm{wt} \%$ under pressure and temperature conditions of $37 \mathrm{~atm}$ and $300 \mathrm{~K}$, respectively. This value is attributed to the polarization of the sample surface (induced by the presence of maghemite crystals). As the iron content exceeded $14 \mathrm{wt} \%$, the maghemite formed larger grains that were poorly dispersed across the surface, thereby causing a decrease in the gravimetric hydrogen adsorption rate. Our experimental results revealed that higher hydrogen storage capacities could be achieved by carefully controlling the dispersion of iron oxides in CNP samples in order to obtain a homogeneously polarized CNP surface. Thus, the hydrogen storage capacity of $\mathrm{Fe} / \mathrm{CNP}$ samples can be significantly increased by appropriately adjusting the surface polarities of the CNP and ensuring well dispersed iron oxide crystals on the surface.

\section{Acknowledgment}

This work was supported in part by the institute of Taiwan National Science Council under Contract no. NSC 101-2221E-008-014.

\section{References}

[1] S. J. Park, B. J. Kim, Y. S. Lee, and M. J. Cho, "Influence of copper electroplating on high pressure hydrogen-storage behaviors of activated carbon fibers," International Journal of Hydrogen Energy, vol. 33, no. 6, pp. 1706-1710, 2008.

[2] M. A. de la Casa-Lillo, F. Lamari-Darkrim, D. Cazorla-Amorós, and A. Linares-Solano, "Hydrogen storage in activated carbons and activated carbon fibers," Journal of Physical Chemistry B, vol. 106, no. 42, pp. 10930-10934, 2002.

[3] M. Rzepka, P. Lamp, and M. A. de la Casa-Lillo, "Physisorption of hydrogen on microporous carbon and carbon nanotubes," Journal of Physical Chemistry B, vol. 102, no. 52, pp. 1089410898, 1998.

[4] P. Larsson, C. M. Araújo, J. A. Larsson, P. Jena, and R. Ahuja, "Role of catalysts in dehydrogenation of $\mathrm{MgH}_{2}$ nanoclusters," Proceedings of the National Academy of Sciences of the United States of America, vol. 105, no. 24, pp. 8227-8231, 2008.

[5] P. C. M. M. Magusin, W. P. Kalisvaart, P. H. L. Notten, and R. A. van Santen, "Hydrogen sites and dynamics in light-weight hydrogen-storage material magnesium-scandium hydride investigated with ${ }^{1} \mathrm{H}$ and ${ }^{2} \mathrm{H}$ NMR," Chemical Physics Letters, vol. 456, no. 1-3, pp. 55-58, 2008.

[6] H. Kabbour, T. F. Baumann, J. H. Satcher, A. Saulnier, and C. C. Ahn, "Toward new candidates for hydrogen storage: highsurface-area carbon aerogels," Chemistry of Materials, vol. 18, no. 26, pp. 6085-6087, 2006.

[7] X. Wu, Y. Gao, and X. C. Zeng, "Hydrogen storage in pillared Li-dispersed boron carbide nanotubes," Journal of Physical Chemistry C, vol. 112, no. 22, pp. 8458-8463, 2008.

[8] S. Kocabas, T. Kopac, G. Dogu, and T. Dogu, "Effect of thermal treatments and palladium loading on hydrogen sorption characteristics of single-walled carbon nanotubes," International Journal of Hydrogen Energy, vol. 33, no. 6, pp. 1693-1699, 2008.

[9] M. Zieliński, R. Wojcieszak, S. Monteverdi, M. Mercy, and M. M. Bettahar, "Hydrogen storage in nickel catalysts supported on activated carbon," International Journal of Hydrogen Energy, vol. 32, no. 8, pp. 1024-1032, 2007.

[10] R. Zacharia, K. Y. Kim, A. K. M. F. Kibriab, and K. S. Nahm, "Enhancement of hydrogen storage capacity of carbon nanotubes via spill-over from vanadium and palladium nanoparticles," Chemical Physics Letters, vol. 412, no. 4-6, pp. 369-375, 2005.

[11] H. S. Kim, H. Lee, K. S. Han et al., "Hydrogen storage in $\mathrm{Ni}$ nanoparticle-dispersed multiwalled carbon nanotubes," Journal of Physical Chemistry B, vol. 109, no. 18, pp. 8983-8986, 2005.

[12] R. M. Cornell and U. Schwertmann, The Iron Oxides: Structure, Properties, Reactions, Occurrences and Uses, Wiley-VCH, 2nd edition, 2003.

[13] F. . Rouquerol, J. Rouquerol, and K. S. W. Sing, Adsorption By Powders and Porous Solids: Principles, Methodology and Applications, Academic Press, 1999.

[14] D. F. Evans and H. Wennerstrom, The Colloidal Domain: Where Physics, Chemistry, Biology and Technology Meet, Wiley-VCH, 2nd edition, 1999.

[15] Y. Li and R. T. Yang, "Hydrogen storage in metal-organic frameworks by bridged hydrogen spillover," Journal of the American Chemical Society, vol. 128, no. 25, pp. 8136-8137, 2006. 

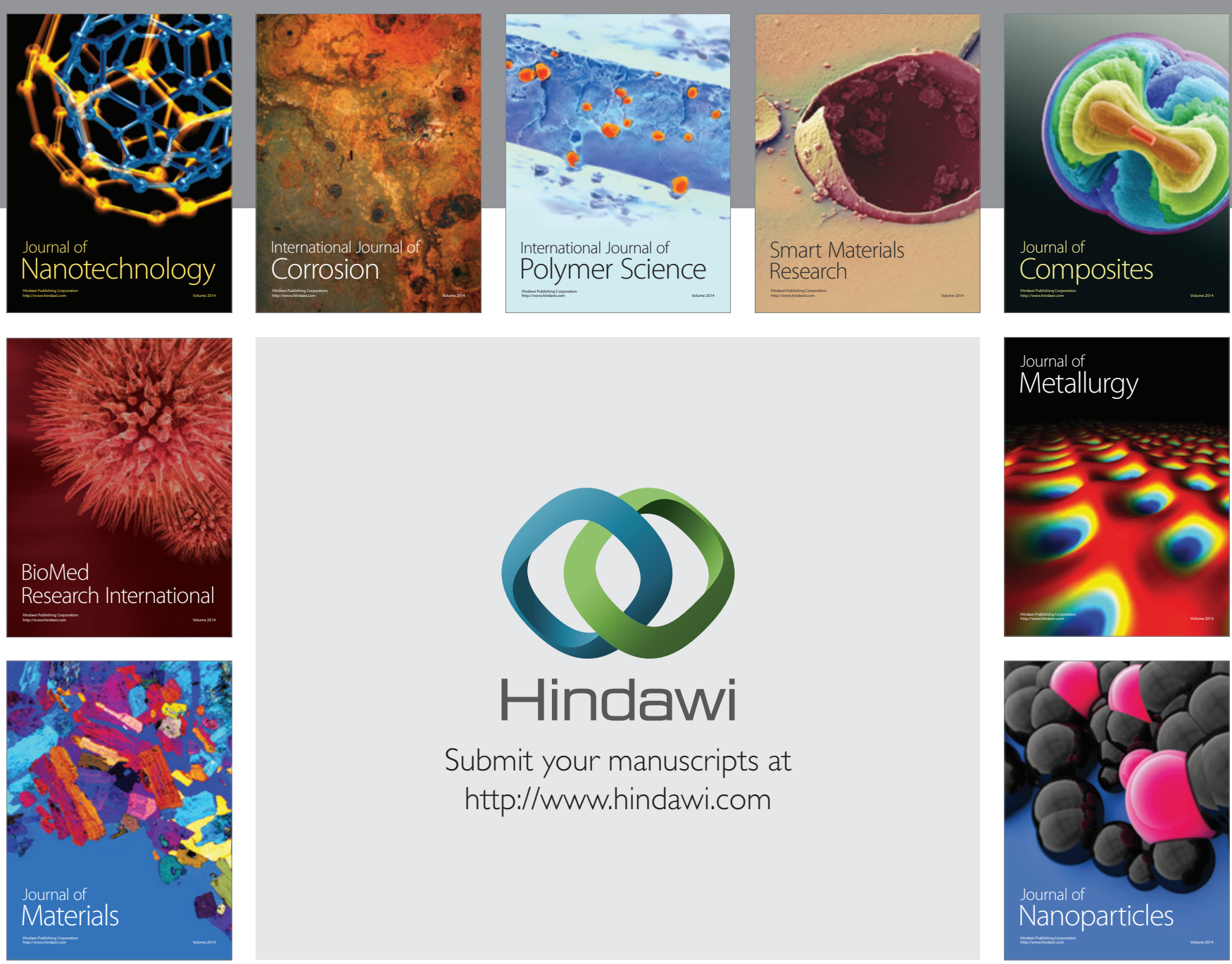

Submit your manuscripts at http://www.hindawi.com
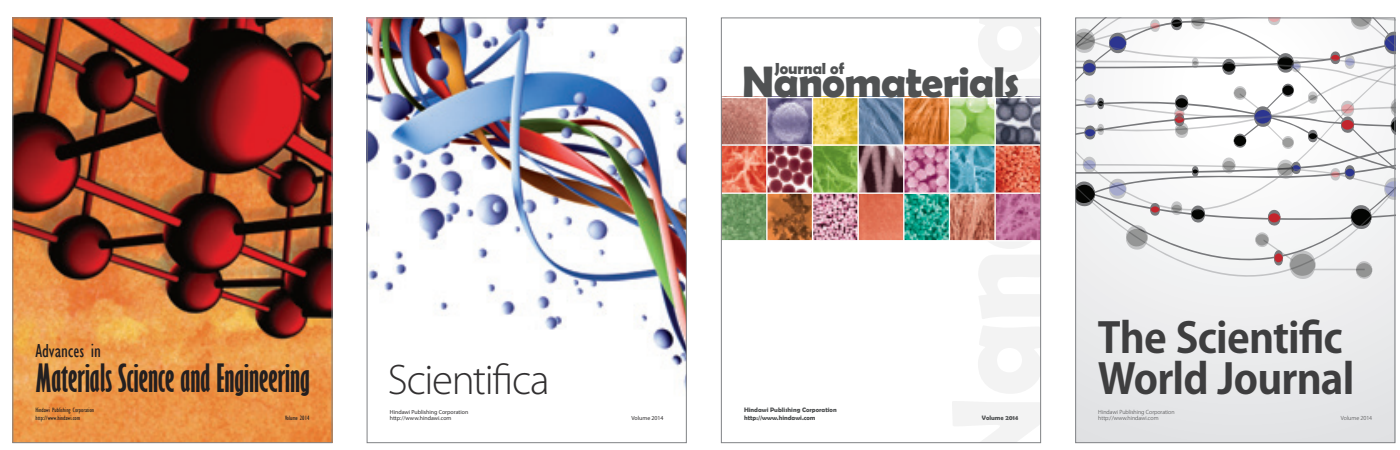

\section{The Scientific World Journal}
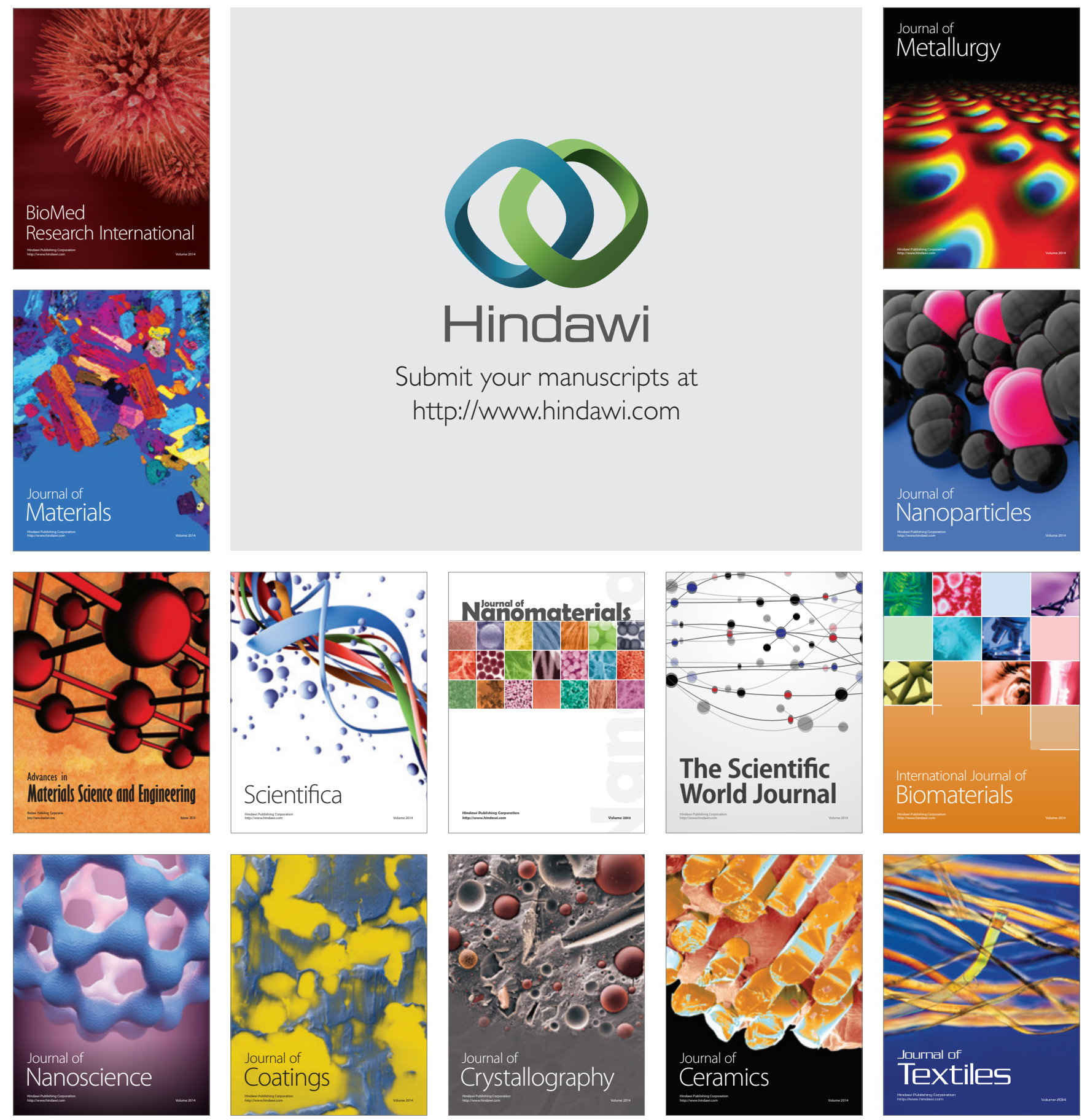